\title{
Optical nanosensor technology - from basic research to industrial applications
}

\author{
Manuela Reitzig ${ }^{1}$, Julia Katzmann ${ }^{1}$, Christiane Schuster ${ }^{1}$, and Thomas Härtling ${ }^{1,2}$ \\ ${ }^{1}$ Fraunhofer Institute for Ceramic Technologies and Systems, \\ Branch Materials Diagnostics (IKTS-MD), Maria-Reiche-Str. 2, 01109 Dresden, Germany \\ Institute for Solid State Electronics, Technische Universität Dresden, 01062 Dresden, Germany
}

\begin{abstract}
:
Optically active nanomaterials enable exciting novel applications of optical measurement technology such as gas sensing with nanoscale infrared antennas, dosimetry with ceramic phosphors, or molecular sensing and detection by means of nanostructured surfaces in general, to name a few only. Some techniques are still in their infancy, while others already led to industrially relevant applications. This article intentionally illustrates both ends of the readiness level scale to provide an idea of the bandwidth currently found in this field of research. On the one hand, surface-enhanced infrared spectroscopy and the fabrication of the necessary infrared nanoantennas as pursued in the group of the authors is described to give an example of an extremely promising, yet not market-ready optical nanosensor technology. On the other hand, the use of ceramic phosphors for radiation dose measurements is presented as a technique with a high technology readiness level and economically relevant application scenarios.
\end{abstract}

Key words: optical sensors, nanomaterials, surface plasmons, ceramic phosphors.

\section{Introduction}

Optical nanosensors consist of nanoscale or nanostructured sensor materials which show a distinct reaction to electromagnetic excitation at optical frequencies. Two major working principles can be distinguished. On the one hand, nanoscale materials can influence direct light-matter interactions such as absorption or scattering events by locally increasing or decreasing the respective interaction cross section. On the other hand, the optical properties of the nanoscale sensor material itself can change due to conditions or processes occurring in the vicinity of the sensor material. The change of the optical properties can then be interrogated in a contact-free fashion from the macroworld.

At Fraunhofer IKTS-MD, optical nanosensor technology is pursued for industrial applications according to the following scheme: optically active nanomaterials are applied at the surface or are incorporated into an object under investigation. By locally enhancing light-matter interactions or by directly changing their optical properties they provide information on material states in a technical component or on a process parameter. Hence, such nanoscale sensor materials can be used for quality assurance and process control in industrial environments.
This article focusses on two examples of optical nanosensor approaches with different technology readiness level. The manipulation of light-matter interactions is illustrated using the example of metal nanoantennas which enhance the absorption of infrared light in organic molecules. Thus, these structures can be used to enhance the signal in infrared absorption spectroscopy. Ample laboratory-based studies were successfully conducted with several kinds of antenna structures, but no market-ready system has surfaced yet. The central challenge here is a cheap, reliable, and reproducible production of the sensor material, i.e. the nanoantennas. One approach to solve this restriction based on ceramic templates is presented in section 2.

The other nanosensor approach discussed in this article is the use of ceramic phosphors. This class of material shows frequency up- or downconversion of absorbed photons. The emission properties change sizeably if the material experienced an exposure to energy, e.g. heating or high-energy radiation. The latter effect has being studied intensively in our group and led to a demonstrator for dosimetry applications for low electron beam doses. As the material and its handling are well understood, ceramic phosphor nanosensors represent a technology that is close to 
introduction on the market. The approach is described in detail in section 3 .

\section{Nanoantennas for infrared spectroscopy}

Infrared (IR) spectroscopy is a powerful tool in material and life sciences as well as in industrial applications for the characterization of molecular species. Based on their unique vibrational bands (so called vibrational fingerprint) molecular species can be unambiguously identified [1]. Thus, IR spectroscopy is in general perfectly suited for various sensing applications such as food control, security, explosive detection, or environmental monitoring. The sensitivity, however, is limited by the molecules IR absorption cross section.

A way to increase the low absorption signals is to exploit surface-enhanced infrared spectroscopy (SEIRS) in which the molecules under investigation are exposed to the enhanced electromagnetic (optical) near-fields around IR nanoantennas, which increases their vibrational signal. To this end, the organic material to be analysed is brought into contact with a nanostructured metal surface (typically gold), which locally enhances the impinging infrared radiation in so-called "hot spots". The enhancement effect is based on the excitation of collective electron oscillations in the metal (surface plasmon polaritons) [2]. Organic molecules located close to such a hot spot can absorb more radiation than unperturbed molecules, i.e. they can remove a higher amount of energy from a ray of infrared light in a transmission experiment [3]. As a result, they can be detected and identified at very low concentrations, as the authors were able to demonstrate e.g. in [4] in cooperation with the Pucci group in Heidelberg.

In the last years, different types of nanostructures for SEIRS have been reported. Initially, randomly arranged metal island films were used which allowed a vibrational signal enhancement by a factor of 2000 [5, 6]. Wetchemically prepared nanoantennas led to 10.000-fold increase of the signal [7]. If the plasmon resonance of the structure spectrally matches a certain IR vibration of the molecule under investigation, an enhancement factor of 500.000 corresponding to attomolar sensitivity can be achieved [8].

Even further enhancement of SEIRS signals can be achieved by exploiting the extraordinary near-field enhancement that occurs if two nanoantennas interact with each other across a very small gap ( $\mathrm{nm}$ range) between their tip ends [9]. This situation is shown in Fig. 1 which depicts the near-field distribution around a
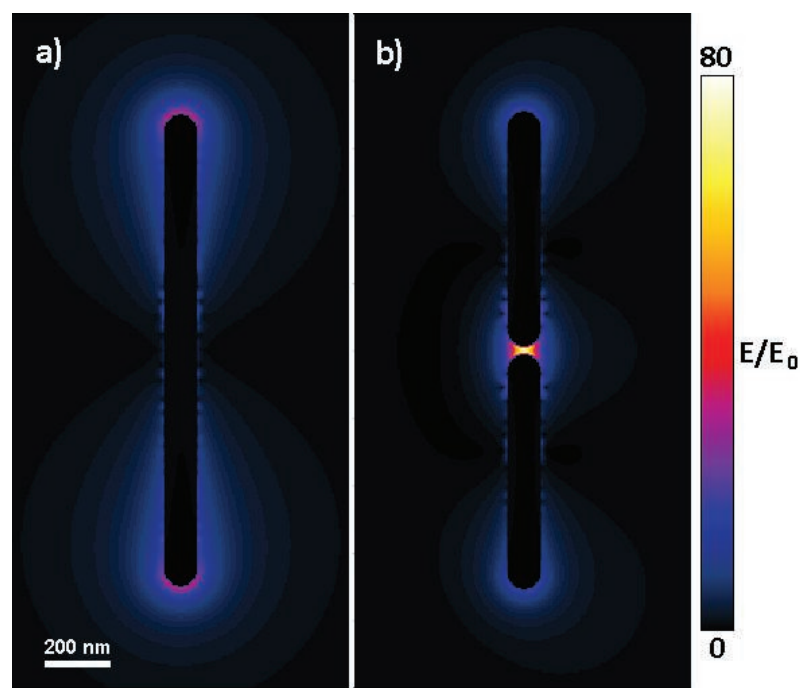

Fig. 1: Enhancement of the electric field (electric field $E$ normalized by impinging field $E_{0}$ ) in the vicinity of a gold IR nanoantenna. a) Single-arm antenna (plasmonic resonance at $\lambda=5,4 \mu \mathrm{m}$ ) and b) two-arm antenna with nanosized gap (gap size $20 \mathrm{~nm}$; plasmonic resonance at $\lambda=3,6 \mu \mathrm{m})$. Calculation performed with the multiple multipole method (MMP) [16].

single gold nanoantenna compared to a narrowspaced pair of antennas with the same overall length. Clearly, the highest field enhancement occurs in the very narrow gap between the antennas, which is then also the source of the highest absorption signal.

The working principle of gap-based signal enhancement was again demonstrated in laboratory experiments [10]. The central challenge that still hampers a broad dissemination and application of SEIRS, e.g. in form of signal-enhancing substrates for ordinary IR spectrometers, is the reliable preparation of such nanosized gaps in a parallel, cheap process suited for centimeter-scale antenna arrays.

In order to cope with this challenge we adapted the template-based nanoantenna fabrication approach reported in [11]. Nanoporous anodized aluminum oxide (AAO) is used as a ceramic matrix material into which metal (Au or $\mathrm{Ag}$ ) is deposited in form of antennas. In an electrochemical setup, aluminum foils can be anodized in a highly controlled way [12 and references therein] which results in pores perpendicular to the substrate surface with diameters ranging from ten to several hundred nanometers and pore distances from 30 to several hundred nanometers. AAO is stable against temperature and most chemicals, allows easy and cheap fabrication and is transparent up to $6 \mu \mathrm{m}$ wavelength. The template geometry can be chosen by adjusting 
the anodization process parameters. A typical AAO template is depicted in Fig. 2.

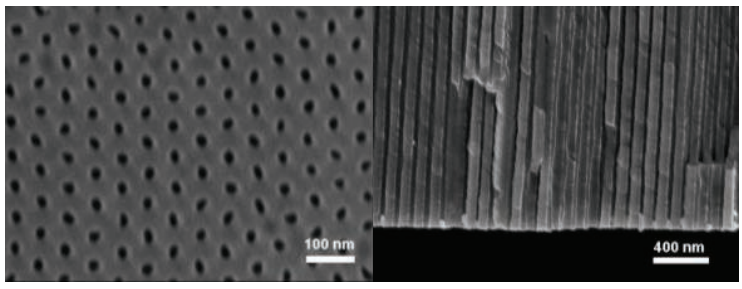

Fig. 2: Porous anodized aluminum oxide forms the microenvironment for the nanoscale IR antennas. (SEM images: left: top view, right: side view). The vertical channels can be electrochemically filled with hollow metal tubes.

After applying a metal layer to one side of the AAO matrix its pores can be filled with metals again in an electrochemical bath. The generation of IR antennas with nanoscale segments was achieved in the following way: in a first step, antennas comprising three segments (Au-Ag-Au) were fabricated. To this end, an electrolyte containing a $\left[\mathrm{Au}\left(\mathrm{S}_{2} \mathrm{O}_{3}\right)\right]^{-3}$ complex was used for gold deposition. The electrochemical cell was then rinsed and a silver nitrate solution was applied for silver precipitation. Finally, again the gold salt electrolyte was used to produce the third antenna segment. The silver segment had a thickness of a few nanometers only as verified by transferring the antennas to a planar substrate, removing of the silver segment by etching with $\mathrm{HNO}_{3}$ and subsequent SEM and TEM imaging (see Fig. 3).

The micrograph images reveal that this approach leads to segmented antennas. However, a complete removal of the silver segment was not achieved so far. The most likely reason for this observation is the interdiffusion of gold and silver during the deposition process. Hence, very small gaps in the regime of several nanometers only as required for efficient SEIRS will presumably require other materials to be deposited. However, we consider the technique as highly promising to reach the aim of large-scale nanoantenna fabrication.
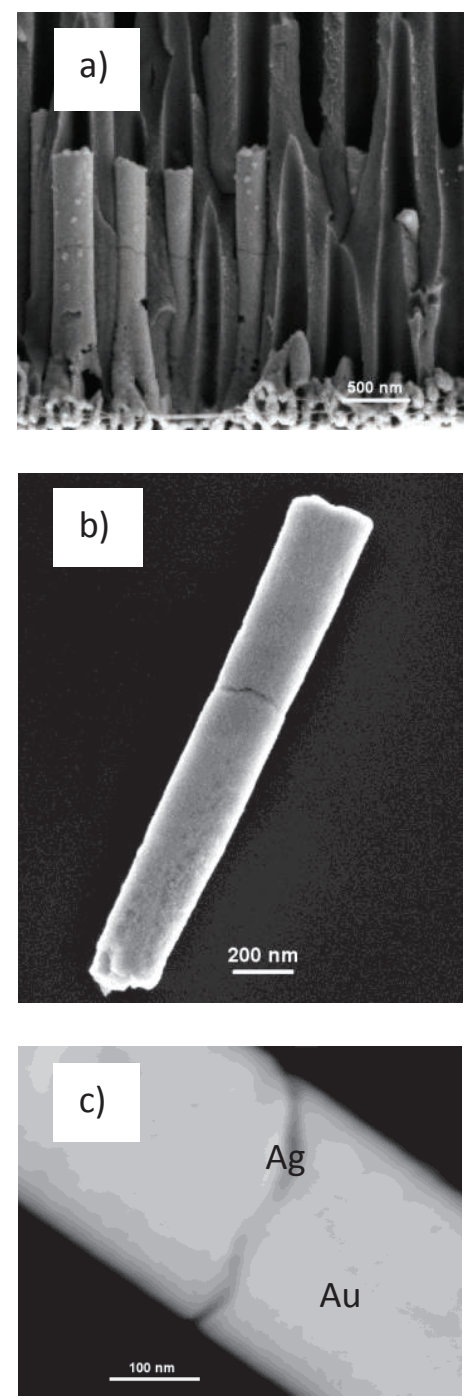

Fig. 3: Nanoscale gaps (or thin silver segments): a) SEM image after cleaving the AAO template and etching of the silver segment by means of $\mathrm{HNO}_{3}$ (b) SEM image of an individual antenna after dissolving the AAO-template with $\mathrm{NaOH}$, transfer of the antenna dispersion onto a silicon wafer and silver etching. (c) TEM material contrast image after transfer of the antenna dispersion onto a TEM grid.

\section{Ceramic phosphors for dosimetry}

In order to illuminate also the other end of the scale, i.e., a high technology readiness level, the example of ceramic phosphors is described in this section. These optical nanosensor materials show a pronounced luminescence emission as response to optical excitation, in particular to laser irradiation. Of special interest is the case of the so-called upconversion phosphors. In this class of materials the interplay between a host crystal lattice and several doping elements enables the subsequent absorption of two low-energy photons and the emission of one high-energy photon [13]. Hence, these phosphors have the 
capability to convert infrared radiation into visible light (see Fig. 4).

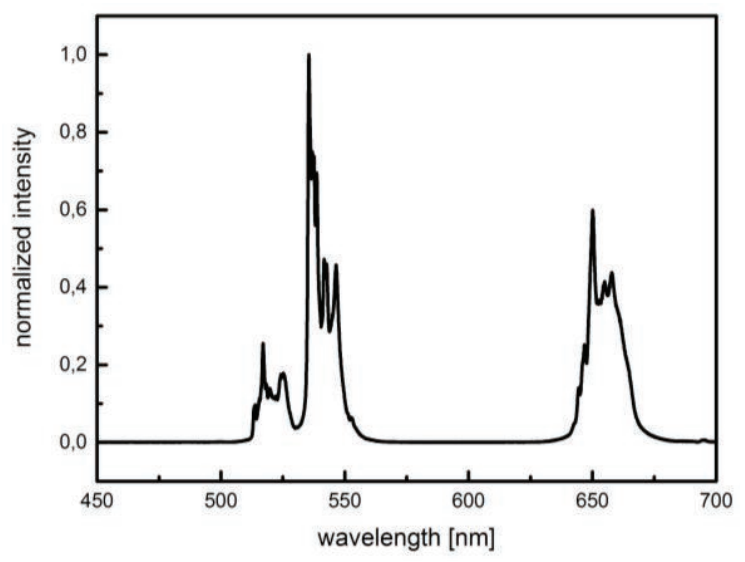

Fig. 4. Upconversion emission spectrum of the applied phosphor after $980 \mathrm{~nm}$ laser excitation.

Besides their intensive light emission the materials show an extraordinary robustness. Most of them are oxides, oxysulfides, or fluorides and are thus inert at high temperatures, insensitive to humidity, and compatible with numerous matrix materials such as inks and pastes. Typically, particles of a size of $0,01-1 \mu \mathrm{m}$ are used so that thin surface coatings or interlayers with integrated particles can be fabricated. It is this robustness and the possibility of large-scale fabrication in the $\mathrm{kg}$ regime which enables their straightforward application for optical nanosensor technology.

For sensing applications, the sensitivity of the phosphor emission decay time to energy exposure can be exploited. Fig. 5 displays the example of the the luminescence decay time $\tau$ after short laser pulse excitation of sodium yttrium oxide $\left(\mathrm{NaYF}_{4}\right.$ : $(\mathrm{Er}, \mathrm{Yb})$, as purchased from Honeywell). In our investigation, the material was exposed to different doses of low energy electron irradiation (150kV acceleration voltage). The graph demonstrates the dependence of the luminescence decay time on the electron dose - it sizeably decreases with increasing dose.

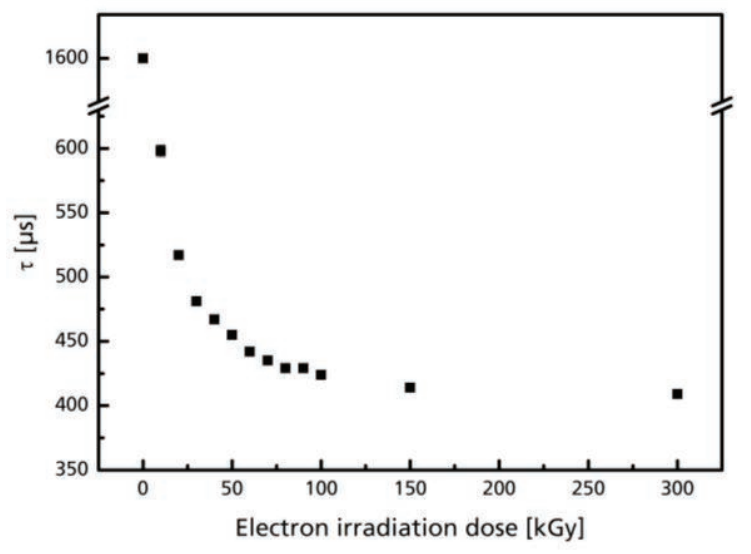

Fig. 5. Luminescence lifetime of the ceramic phosphor emission after exposure to electron beam irradiation of varying dose. A clear decay of the luminescence lifetime with increasing dose is observable.

This distinct material property can for example be applied for determining the irradiation dose during electron beam sterilization on arbitrarily formed surfaces and complicated accessibility, e.g. of medical products, their packages, or in food packaging. The use of commercially available dosimeter stripes is often not possible in these cases. Using this material, dosimetrically active packages and foil stripes were developed at IKTS-MD by physically incorporating upconversion phosphors into polymer foils [14]. Integrating the ceramic nanosensors into a packaging material allows a proof of surface sterilization directly during the sterilization process, e.g. during or directly after the irradiation of a packaged medical product. Details of the testing and read-out process are described in [15]. Furthermore, validation and revalidation of electron beam irradiation systems can be carried out fast, reliably, and contact-free using such dosimeter stripes.

\section{Summary}

The two technology examples described in this article were intentionally chosen to reflect that there is a broad bandwidth in terms of readiness level for optical nanosensor technology. Concerning surface enhanced infrared spectroscopy, our results as well as those reported in the literature demonstrate that the technique is extremely promising, but still lacks reproducibility. The authors consider the nanoantenna fabrication aspect the most pressing task for the scientific community in order to bring SEIRS into industrial applications. Once this hurdle is taken, the prospects and potentials of the method are great, a feature SEIRS has in common with many other 
nanomaterial-based optical sensing approaches.

The motivation for further pushing the limits of several nanosensor technologies is high, especially as successful examples show a sizeable impact and economic relevance. Dosimetry exploiting the optical emission features of ceramic phosphors was chosen as an example in this article. The successful integration of such phosphors into polymer foils enables monitoring and controlling industrial irradiation processes, applications intensively pursued at Fraunhofer IKTS-MD.

\section{Acknowledgement}

The authors thank the DFG Research Training Group 1401 and the Fraunhofer Internal programs (Grant Attract 692271 and SteriHealth 600075) for funding.

\section{References}

[1] B. Stuart, Infrared spectroscopy: Fundamentals and applications. Analytical techniques in the sciences, Wiley (2004).

[2] U. Kreibig und M. Vollmer: Optical Properties of Metal Clusters. Springer, Berlin; Heidelberg, (2010).

[3] F. Neubrech, A. Pucci, T. Cornelius, S. Karim, A. García-Etxarri, und J. Aizpurua: Resonant plasmonic and vibrational coupling in a tailored nanoantenna for infrared detection. Phys. Rev. Lett. 101, 157403 (2008).

[4] C. Huck, F. Neubrech, J. Vogt, A. Toma, D. Gerbert, J. Katzmann, T. Härtling, A. Pucci, Surface-Enhanced Infrared Spectroscopy Using Nanometer-SizedGaps. ACS Nano 8, 4908 (2014).

[5] D. Enders and A. Pucci. Surface enhanced infrared absorption of octadecanethiol on wetchemically prepared Au nanoparticle films. Applied Physics Letters, 88(18):184104 (2006).

[6] K. Ataka, and J. Heberle, Biochemical applications of surface-enhanced infrared absorption spectroscopy, Anal. Bioanal. Chem. 388:47-54 (2007).

[7] F. Le et al., Metallic nanoparticle arrays: a common substrate for both surface-enhanced Raman scattering and surface-enhanced infrared absorption, ACS Nano, 2 (4), 707-718, (2008)

[8] F. Neubrech, et al., Giant infrared signals from molecular vibrations by the action of a tailored nanoantenna, Phys. Rev. Lett. 101, 157403 (2008).

[9] D. Weber, J. Katzmann, F. Neubrech, T. Härtling, and A. Pucci, Spectral tuning of IR-resonant nanoantennas by nanogap engineering. Opt. Mater. Expr. 1, 1301 (2011).
[10] F. Neubrech, D. Weber, J. Katzmann, C. Huck, A. Toma, E. Di Fabrizio, A. Pucci, and T. Härtling, Infrared optical properties of nanoantenna dimers with photochemically narrowed gaps in the $5 \mathrm{~nm}$ regime. ACS Nano 6, 7326 (2012).

[11] L. Qin, S. Park, L. Huang, und C. A. Mirkin: Onwire lithography. Science 309, 113 (2005).

[12] J. Katzmann, Untersuchung zur effizienten Herstellung von Substraten für die oberflächenverstärkte Infrarotspektroskopie. Dissertation, TU Dresden (2015).

[13] M. P. Hehlen, M. L. F. Phillips, N.J. Cockroft, H. U. Güdel, Upconversion Phosphors, Encyclopedia of Materials: Science and Technology, Burschow, K. H. J., Ed., Pergamon/Elsevier Science Ltd., New York, Oxford, 9456 (2001).

[14] M. Reitzig, M. Winkler, T. Härtling, O. Röder, J. Opitz, Evaluation of low energy electron beam dose application by means of a portable optical device. Optical Engineering 53, 114102 (2014).

[15] M. Reitzig et al., Manuscript in preparation.

[16] Hafner, C. Electrical Engineering (Archiv für Elektrotechnik) 69, 321-325 (1986). 